Marcelo Motta Veiga ${ }^{1}$

Francisco José de Castro Moura Duarte $^{2}$

Luiz Antonio Meirelles ${ }^{2}$

Alain Garrigou ${ }^{3}$

Isabelle Baldi ${ }^{4}$

\section{A contaminação por agrotóxicos e os Equipamentos de Proteção Individual (EPIs)}

\author{
Contamination by pesticides and Personal Protective Equipment
}

(PPE)
${ }^{1}$ Departamento de Saneamento e Saúde Ambiental, Escola Nacional de Saúde Pública, Fundação Oswaldo Cruz. Ministério da Saúde. Rio de Janeiro.

${ }^{2}$ Programa de Engenharia de Produção, Instituto Alberto Luiz Coimbra de Pós-Graduação e Pesquisa de Engenharia (COPPE), Universidade Federal do Rio de Janeiro. Rio de Janeiro.

${ }^{3}$ LSTE, Dpt. HSE, IUT, Université Bordeaux 1. França.

${ }^{4}$ ISPED, LSTE, case 11, Université Victor Segalen Bordeaux 2, Case 11. França

Contato:

Marcelo Motta Veiga

Departamento de Saneamento e Saúde Ambiental

Escola Nacional de Saúde Pública

Fundação Oswaldo Cruz

Ministério da Saúde

Rua Leopoldo Bulhões, $1480 / 5^{\circ}$

andar-Manguinhos

CEP: 21041-210 - Rio de Janeiro-

$\mathrm{RJ}$ - Brasil

E-mail:

mveiga@ensp.fiocruz.br

\section{Resumo}

Este trabalho analisou a eficiência e a adequação dos Equipamentos de Proteção Individuais (EPIs) utilizados na manipulação e na aplicação de agrotóxicos nas agriculturas brasileira e francesa. Essa análise foi feita a partir de dois casos: o primeiro, na cultura de tomate numa pequena comunidade rural do Brasil e o segundo, numa vinicultura na França. As evidências encontradas mostraram que os EPIs utilizados em ambos os casos, além de não protegerem integralmente o trabalhador contra o agrotóxico, ainda agravaram os riscos e perigos, pois se tornaram fontes de contaminação. Conclui-se que, nos casos analisados, os EPIs não eliminaram nem neutralizaram a insalubridade, conforme estatui a legislação, e ainda aumentaram a probabilidade de contaminação dos trabalhadores rurais em algumas atividades. Discutiu-se, ainda, a possibilidade dos EPIs apresentarem lacunas funcionais no projeto, na concepção, no uso, na manutenção, no armazenamento e no descarte, que devem ser melhor avaliadas através de futuros estudos de desenvolvimento tecnológico.

Palavras-chaves: agrotóxico, Equipamento de Proteção Individual, EPI, contaminação, ergonomia.

\begin{abstract}
This study has analyzed the efficiency and adequacy of Personal Protective Equipment (PPE) used when mixing and spraying pesticides in Brazilian and French farming. Analysis was based on two case studies: The first one, concerning a tomato crop located in a small rural community in the Southeast of Brazil; the second, a vineyard in France. The findings showed that PPE used in both cases has not protected properly against pesticide and has sometimes even turned into a source of contamination. The study concluded that PPE could not eliminate or neutralize unhealthy conditions related to pesticide use, as law demands. Moreover, in some cases, it might have caused rural workers' contamination. It also shows that PPE might not have been correctly planned, conceived, used, maintained, stored and disposed. Theses problems should be analyzed in further technological development studies.
\end{abstract}

Keywords: pesticide, Personal Protective Equipment, PPE, contamination, ergonomics. 


\section{Introdução}

Este trabalho se propõe a discutir o tema da eficiência e da adequação dos Equipamentos de Proteção Individual (EPIs) utilizados na aplicação de agrotóxicos a partir de estudos anteriores realizados na cultura de tomate numa pequena comunidade rural do Brasil (VEIGA et al., 2006) e numa viticultura na França (BALDI et al., 2006).

O emprego de EPIs, apesar de não desejado, deve ser considerado como tecnologia de proteção disponível dentro de uma visão integrada e sistêmica de abordagem dos problemas ocupacionais. A eficiência de todo sistema de Saúde e Segurança no Trabalho (SST) está intimamente relacionada à forma como é conduzida e balanceada, no processo decisório, a escolha das alternativas de prevenção, proteção e controle (ILO, 2001). Contudo, um projeto eficiente de SST deve contemplar, num enfoque sistêmico, a integração de todos os elementos relevantes para estabelecer políticas e estratégias adequadas a cada realidade situacional. A gestão eficaz de um programa de SST deve ambicionar, ainda, aumentos de produtividade nos processos de trabalho com reduções nos riscos.

A Organização Internacional do Trabalho - OIT (International Labour Office - ILO) estabelece que os perigos e riscos à segurança e à saúde dos trabalhadores devem ser identificados e avaliados de forma contínua e que as medidas de prevenção e proteção devem ser implementadas seguindo a seguinte ordem de prioridades (ILO, 2001):

(a) eliminar os riscos/perigos;

(b) controlar as fontes de risco/perigo utilizando técnicas de engenharia e/ou medidas de gestão;

(c) minimizar os riscos/perigos através de projetos de sistemas de segurança, que devem incluir mecanismos de controle gerenciais;

(d) onde houver riscos/perigos residuais que não puderem ser evitados/controlados por medidas coletivas, o empregador deve providenciar os equipamentos de proteção adequados.

Diversos problemas podem acarretar a inadequação dos EPIs a certas condições de trabalho. Algumas das características desejáveis aos EPIs e que foram projetadas para conferir maior segurança podem estar introduzindo dificuldades operacionais em muitas situações de trabalho. Por exemplo, uma maior resistência de um tecido à permeabilidade, uma maior resistência ao choque elétrico, uma maior resistência ao calor podem estar associados a aumento de peso, menor conforto térmico e menos portabilidade dos EPIs. Outro aspecto importante é a dificuldade da adequação dos EPIs às características antropométricas e ambientais de cada localidade.

Nos Estados Unidos, a adequação e a eficiência de equipamentos de proteção foram testadas ao limi- te, quando as situações se tornaram extremas. Após $\mathrm{o}$ ataque terrorista às torres gêmeas em Nova Iorque, em 11 de setembro de 2001, foi possível verificar a inadequação e a ineficiência de diversos tipos de EPIs, que, apesar de projetados para proteger contra agentes específicos, limitavam as ações dos trabalhadores, e.g. eram muito pesados e dificultavam o deslocamento. Essa inadequação e ineficiência dos EPIs foi novamente constatada no verão de 2005, por ocasião do atendimento às vítimas do furacão Katrina em Nova Orleans, principalmente no que diz respeito à exposição ao calor prolongado (estresse térmico) a que foram submetidos os trabalhadores que atuaram no resgate das vítimas (NIEHS, 2005; SARGENT, 2005; STULL \& MACDOUGALL, 2006; NIOSH, 2002, 2003, 2004a e 2006).

Nestes dois acidentes, devem ter ocorrido muitas vítimas desnecessárias relacionadas diretamente ou indiretamente ao uso de EPIs. Essas vítimas, na grande maioria, foram trabalhadores nas operações de resgate que não eram vítimas no momento dos acidentes e tinham a função de mitigar as conseqüências. Com isso, levantou-se a hipótese de que os EPIs que tinham, primordialmente, a função de oferecer maior segurança e saúde aos trabalhadores não estavam cumprindo o seu papel de forma eficiente.

Uma conseqüência direta dessas experiências negativas americanas foi uma significativa alteração nas normas e nos padrões de proteção exigidos com relação à necessidade e ao uso de EPIs nos Estados Unidos. Conseqüentemente, criou-se uma demanda por desenvolvimento em tecnologia de proteção associado ao uso de EPIs. Essa nova realidade compelia o desenvolvimento de tecnologias analíticas (incluindo validação dessas tecnologias) de forma que se pudesse avaliar se os atuais EPIs atendiam a essas novas exigências técnicas e legais, ou seja, necessitou-se de aprimorar o estado da técnica em EPIs (NIOSH, 2004).

Com a finalidade de atender a essa demanda por desenvolvimento tecnológico em EPIs, foi criado, a partir de 2001 (entrando em operação em 2003), pelo Instituto Nacional para Saúde e Segurança Ocupacional (National Institute of Occupational Safety and Health - NIOSH), um laboratório denominado Laboratório Nacional de Tecnologia em Proteção Pessoal (National Personal Protective Technology Laboratory - NPPTL), que hoje se tornou referência mundial em matéria de tecnologia de proteção pessoal (NIOSH, 2004).

Também na França, os EPIs tiveram sua eficiência questionada. Uma recente pesquisa do Ministério da Agricultura francês (MINAG, 2006) colocou em evidência que os equipamentos de proteção recomendados raramente eram utilizados, o que permitiu questionar a eficácia real dos meios de proteção recomendados. Jourdan (1989), Bernon (2002) e Brunet et al. (2005) estudaram as dificuldades li- 
gadas à utilização dos EPIs e as restrições que são geradas por sua utilização.

No Brasil, o problema de inadequação dos EPIs às condições ergonômicas e ambientais também não é estranho. Na agricultura brasileira, especialmente em pequenas comunidades rurais, é comum deparar-se com trabalhadores rurais sem os EPIs obrigatórios durante a manipulação e a aplicação de agrotóxicos. Uma das principais razões para não se utilizar EPIs reside no fato de que muitos dos EPIs utilizados na agricultura, devido a sua inadequação, podem provocar desconforto térmico, tornando-os bastante incômodos para uso, podendo levar, em casos extremos, ao estresse térmico do trabalhador rural (COUTINHO et al., 1994).

Apesar da utilização de EPIs poder resultar num problema para a saúde dos trabalhadores, não foi identificado laboratório independente no Brasil que esteja analisando a adequação das tecnologias de EPIs. Os laboratórios de EPIs existentes no Brasil credenciados pelo Ministério do Trabalho e Emprego (MTE) se limitam a realizar testes padrões que visam principalmente aprovar (obtenção do certificado de aprovação) a comercialização e/ou produção de certos EPIs no Brasil.

Porém, a maioria dos EPIs comercializados no Brasil não passa por qualquer teste por falta de laboratórios capacitados/credenciados para realizar as análises necessárias. Não existem sequer padrões para conforto térmico ou para permeabilidade. Com isso, muitos EPIs são comercializados no Brasil apenas com a assinatura de um termo de responsabilidade.

\section{Agrotóxicos e EPIs na Legislação Brasileira}

Este estudo escolheu a legislação brasileira para exemplificar a expectativa legal com a utilização de EPIs. A legislação brasileira sobre EPI é a Norma Regulamentadora $\mathrm{n}^{\mathrm{o}} 6$ (NR-6) ${ }^{5}$. A NR-6 considera EPI todo dispositivo ou produto, de uso individual utilizado pelo trabalhador, destinado à proteção de riscos suscetíveis de ameaçar a segurança e a saúde no trabalho.

A NR-6 condiciona o uso e a comercialização de EPI à obtenção de um Certificado de Aprovação (CA) expedido pelo Ministério do Trabalho e Emprego e define os procedimentos para o fabricante interessado em comercializar EPI.

A norma ainda define como competência do empregador (no que se refere ao uso de EPI): adquirir o EPI adequado ao risco de cada atividade; exigir seu uso; fornecer ao trabalhador somente o EPI aprovado pelo órgão nacional competente em matéria de segurança e saúde no trabalho; orientar e treinar o trabalhador sobre o uso adequado, a guarda e a conservação; substituir imediatamente quando danificado ou extraviado; responsabilizar-se pela higienização e manutenção periódica; e comunicar ao MTE qualquer irregularidade observada.

Quanto ao empregado: compete a este usar o EPI apenas para a finalidade a que se destina; responsabilizar-se pela guarda e conservação; comunicar ao empregador qualquer alteração que o torne impróprio para uso; e cumprir as determinações do empregador sobre o uso adequado.

A legislação brasileira que define as atividades ou operações consideradas insalubres é a Norma Regulamentadora $n^{\circ} 15$ (NR-15) ${ }^{6}$. Nesta norma são consideradas insalubres as atividades que sejam desenvolvidas:

15.1.1 Acima dos limites de tolerância (...)

15.1.5 Entende-se por "Limite de Tolerância”, para os fins desta Norma, a concentração ou intensidade máxima ou mínima, relacionada com a natureza e o tempo de exposição ao agente, que não causará dano à saúde do trabalhador, durante a sua vida laboral.

Existem aspectos econômicos e jurídicos importantes em decorrência da NR-15:

15.2 O exercício de trabalho em condições de insalubridade, de acordo com os subitens do item anterior, assegura ao trabalhador a percepção de adicional, incidente sobre o salário mínimo da região, equivalente a:

\section{(...)}

15.4 A eliminação ou neutralização da insalubridade determinará a cessação do pagamento do adicional respectivo.

15.4.1 A eliminação ou neutralização da insalubridade deverá ocorrer:

a) com a adoção de medidas de ordem geral que conservem o ambiente de trabalho dentro dos limites de tolerância;

b) com a utilização de equipamento de proteção individual.

15.4.1.1 Cabe à autoridade regional competente em matéria de segurança e saúde do trabalhador, comprovada a insalubridade por laudo técnico de engenheiro de segurança do trabalho ou médico do trabalho, devidamente habilitado, fixar adicional devido aos empregados expostos à insalubridade quando impraticável sua eliminação ou neutralização.

\footnotetext{
${ }^{5}$ MINISTÉRIO DO TRABALHO E EMPREGO. Portaria 3214, de 08 de junho de 1978. Norma Regulamentadora no 6 (NR-6). Equipamento de proteção individual. Disponível em: http://www.mte.gov.br/legislacao/normas_regulamentadoras/default.asp

${ }^{6}$ MINISTÉRIO DO TRABALHO E EMPREGO. Portaria 3214, de 08 de junho de 1978. Norma Regulamentadora 15 (NR-15). Atividades e Operações Insalubres. Disponível em: http://www.mte.gov.br/legislacao/normas_regulamentadoras/default.asp
} 
15.4.1.2 A eliminação ou neutralização da insalubridade ficará caracterizada através de avaliação pericial por órgão competente, que comprove a inexistência de risco à saúde do trabalhador.

Como fatores econômicos, tem-se que o exercício de trabalho em condições insalubres garante um adicional pecuniário ao trabalhador e ainda representa um aspecto previdenciário, na maioria dos casos, que reduz o tempo de contribuições necessário para a aposentadoria. Como aspecto jurídico, têm-se possíveis ações trabalhistas indenizatórias.

Porém, esta mesma NR-15 deixa uma brecha que permite ao empregador "eliminar ou neutralizar a insalubridade" através da utilização de equipamento de proteção individual. Isso explicaria o fato do uso de EPIs ser a solução mais utilizada pelos empregadores para eliminar ou neutralizar os riscos em detrimento do estabelecido pelo International Labour Office (ILO, 2001).

\section{O equipamento de proteção individual (EPI) como tecnologia em saúde}

O sistema de saúde brasileiro pauta-se pelos princípios constitucionais da universalidade, eqüidade e integralidade. A Política Nacional de Ciência e Tecnologia em Saúde (C\&T/S), como componente da Política Nacional de Saúde, visa integrar os processos de desenvolvimento científico e tecnológico aos serviços de saúde oferecidos à sociedade.

A aplicação destes princípios constitucionais à Política Nacional de Ciência e Tecnologia em Saúde (C\&T/S) deve objetivar a promoção, proteção e prevenção da saúde, contribuindo à redução das desigualdades socioeconômicas existentes no país. Porém, essas iniqüidades se refletem tanto no perfil epidemiológico das classes sociais quanto na distribuição das tecnologias de saúde (MS, 2002; ABRASCO, 2002).

Dentro de uma visão lato sensu, mais abrangente, tecnologias em saúde poderiam ser conceituadas, como equipamentos, medicamentos, insumos e procedimentos utilizados na prestação, organização e infra-estrutura de serviços de saúde.

Então, de acordo com o seu propósito, as tecnologias em saúde poderiam ser classificadas em tecnologias de proteção, prevenção, promoção, assistenciais e de apoio à saúde, em que o equipamento de proteção individual (EPI) se enquadraria como uma tecnologia de proteção.

Oliveira e Machado Neto (2005) avaliaram a segurança no trabalho relacionada com o uso de EPIs na aplicação de agrotóxicos para a cultura da batata. Eles constataram que o tipo de EPI utilizado influenciava diretamente na possibilidade de exposição dos trabalhadores rurais e, ainda, que mesmo utilizando os EPIs recomendados, os trabalhadores rurais continuavam se contaminando, uma vez que os EPIs foram "erroneamente recomendados com base apenas na classe toxicológica e não na exposição ocupacional que as condições de trabalho propiciam e na sua distribuição pelo corpo do trabalhador", ou seja, inadequados para situação real encontrada.

Por isso, faz-se necessário avaliar a adequação de cada tecnologia em saúde (ATS) e as condições ambientais e antropométricas encontradas em cada situação de fato. No caso deste estudo, os EPIs utilizados na aplicação de agrotóxicos seriam essa tecnologia de proteção. A avaliação de tecnologias em saúde seria uma das ferramentas mais importantes à gestão de um sistema de saúde eficiente, tendo implicações sociais, éticas, legais e econômicas (EDUARDO \& ISAURA, 1998).

Essa avaliação de tecnologias em saúde (ATS) se trataria de uma pesquisa complexa, devendo incluir um levantamento sistemático de informações sobre o estado da arte e da técnica, uma análise socioeconômica e testes empíricos da tecnologia que está sendo avaliada.

Por isso, a avaliação de uma tecnologia em saúde tentaria identificar evidências científicas de efetividade, de custo e de risco do seu uso e não uso, visando à adequação, seleção, aquisição, distribuição ou uso apropriado dessa tecnologia, incluindo a sua real necessidade (MS, 2002; ABRASCO, 2002).

\section{Conforto Térmico}

A legislação em muitos países da Europa já exige que sejam avaliados os riscos associados à utilização de Equipamentos de Proteção Individual (EPI). Entre esses riscos estariam os prejuízos à saúde ocasionados pela hipertermia. Além, dos problemas para saúde humana, a falta de conforto térmico no ambiente de trabalho, provocado pelo uso dos EPIs, acaba por ter efeitos, também, econômicos, uma vez que influi diretamente na produtividade e na qualidade do trabalho realizado (CROCKFORD, 1999).

Um conceito para conforto térmico seria sensação (subjetiva) de bem-estar experimentada por uma pessoa como resultado da combinação satisfatória das variáveis temperatura radiante média, umidade relativa, temperatura ambiente e velocidade relativa do ar (FUNDACENTRO, 1999).

A fisiologia térmica do corpo humano é bastante complexa, mas pode-se dizer que funciona como um sistema termodinâmico que se termorregula tentando manter a temperatura interna próxima dos $37^{\circ} \mathrm{C}$. O conforto térmico estaria, então, associado à dificuldade do corpo humano em perder ou reter calor para manter este equilíbrio térmico termorregulador (FUNDACENTRO, 2001).

As principais variáveis que influenciariam nesta termorregulação podem ser dividas em ambientais (temperatura radiante média, temperatura do ar, umidade relativa do ar, ventilação do ambiente) e pessoais (metabolismo, vestimenta utilizada e tipo de atividade 
realizada). Este balanço térmico, que se dá entre a taxa de calor produzido pelo corpo (taxa de metabolismo menos trabalho realizado) e o calor cedido ao ambiente pela pele (calor latente e calor sensível) e pela respiração, é vital para vida humana (FUNDACENTRO, 2001).

Contudo, apesar de necessária a muitas atividades laborais, a utilização de equipamento de proteção individual influencia neste equilíbrio térmico por dificultar a perda de calor pelo corpo humano por convecção, radiação e evaporação. Existiria retenção de calor toda vez que o corpo humano não conseguisse perder calor na quantidade necessária devido ao isolamento térmico provocado pela utilização de EPIs, resultando num conseqüente aumento na temperatura corporal. Por isso, em atividades em que a utilização de EPIs seja essencial, existiria uma probabilidade maior de problemas para saúde provocados pelo calor. Estima-se que a probabilidade de se apresentar uma doença relacionada ao calor excessivo seria de um em cada mil trabalhadores que utilizam EPIs sob essa condição, por ano trabalhado (CROCKFORD, 1999).

Por isso, seria correto afirmar que a temperatura do ambiente afetaria o desempenho profissional. Temperaturas corporais além dos limites ótimos $\left(36,5^{\circ}-37,5^{\circ}\right.$ C) afetariam o desempenho físico e mental, podendo levar a comprometimentos fisiológicos e patológicos. O corpo humano, não conseguindo manter o equilíbrio térmico, aumentaria a taxa de retenção de calor influenciando na eficiência e na produtividade dos trabalhadores (AHASAN et al., 2002; RODAHL, 2003).

Porém, os limites estabelecidos nas legislações para conforto térmico e temperaturas extremas estão baseados nas reações agudas de trabalhadores expostos ao calor e não nos seus efeitos crônicos. Por isso, podese dizer que a literatura sobre a exposição contínua e prolongada de trabalhadores ao calor ainda carece de futuros estudos (WOOD, 2004).

Outro agravante para o caso da utilização de EPIs por trabalhadores rurais em países de clima quente seria o fato de que a realização de atividades profissionais em localidades de clima quente e úmido seria mais insalubre do que as mesmas atividades realizadas em condições mais amenas. Um trabalhador executando uma atividade moderada sob condições amenas, utilizando roupas leves, levaria em média 90 minutos para elevar em $1,5^{\circ} \mathrm{C}$ sua temperatura corporal. Caso este mesmo trabalhador utilizasse uma roupa impermeável e sintética, esse tempo cairia para 20 minutos. Com isso, o tipo de EPI, junto com as condições ambientais, influenciam no tempo limite que um trabalhador poderia estar exposto a essas condições ambientais dentro da faixa do conforto térmico (HAVENITH, 1999).

\section{Estudos considerados}

Os EPIs são projetados, no caso de agrotóxicos, de forma a garantir proteção contra agentes quími- cos externos, ou seja, para manter certas substâncias "fora" do organismo. As mesmas propriedades físicas e químicas que fornecem aos EPIs essa característica de proteção também os transformam, freqüentemente, em bastante desconfortáveis e/ou inadequados. Esse desconforto no uso pode tornar a utilização de alguns EPIs um verdadeiro incômodo.

Os EPIs podem, ainda, se tornar uma fonte de contaminação, criando um risco à saúde humana. Nos dois casos apresentados a seguir, as falhas nos cuidados básicos de conservação e nos procedimentos de despir/vestir colocam os EPIs como prováveis fontes de contaminação do trabalhador.

\section{A contaminação por agrotóxicos na cultura do tomate}

Num estudo recentemente publicado, mostrou-se a contaminação ambiental por agrotóxicos na região da cultura do tomate numa pequena comunidade rural do Estado do Rio de Janeiro. Neste estudo, selecionouse 27 pontos de coleta, sendo 20 de sistemas hídricos superficiais e 7 de sistemas hídricos subterrâneos utilizados para consumo humano (VEIGA et al., 2006).

Para o período de março a setembro de 2003, dos 27 pontos de coleta selecionados, apenas 8 não apresentaram contaminação detectável, sendo que em duas ocasiões estas contaminações ultrapassaram o permitido pela legislação (Conama $n^{\circ} 357 / 05$ ), ou seja, $70 \%$ dos pontos analisados apresentaram contaminação detectável. Esta contaminação por agrotóxicos atingiria potencialmente toda a população local, mas de maneira mais agressiva penalizaria os próprios usuários (trabalhadores rurais).

O indicativo de contaminação de sistemas hídricos por organofosforados e carbamatos utilizados neste estudo foi a atividade da enzima acetilcolinesterase (percentagem de inibição), conforme estabelecido na Portaria $n^{\circ} 518 / 04$, que estabelece os valores máximos das substâncias químicas que representam risco para a saúde. Essa análise de inibição enzimática é utilizada por avaliação indireta em comparação com o paration quimicamente puro da concentração dos compostos organofosforados e carbamatos totais.

Uma pesquisa do IBGE na mesma localidade, em 1990, detectou que a maioria dos trabalhadores rurais (70\%) não costumava usar qualquer tipo de equipamento de proteção individual (EPI) na preparação e no uso de agrotóxicos (COUTINHO et al., 1994).

Resultados preliminares de uma pesquisa realizada com os trabalhadores rurais na região, em 2006, ratificaram a maioria dos resultados dessa pesquisa do IBGE, e detectaram que os trabalhadores, na sua maioria, são jovens (20 a 25 anos) com pouca instrução (1 a 3 anos de educação formal). Observou-se, ainda, que os trabalhadores rurais não seguem as recomendações quanto a manutenção, lavagem, vestir/despir, descarte, armazenagem dos EPIs. Com relação ao uso dos EPIs, a maior reclamação foi o desconforto térmico, 
principalmente em dias quentes. Outra reclamação foi o embaçamento da máscara facial pela respiração durante a aplicação dos agrotóxicos.

A região estudada apresenta um inverno frio e seco e um verão quente e chuvoso. A temperatura média anual gira em torno de $18^{\circ} \mathrm{C}$ a $22^{\circ} \mathrm{C}$. Porém, as temperaturas máximas chegam a ultrapassar os $40^{\circ} \mathrm{C}$ no verão e as mínimas podem chegar próximas de $0^{\circ}$ $\mathrm{C}$ nas partes mais altas, no inverno.

Por isso, o EPI que foi projetado para minimizar a contaminação por agrotóxicos também reduziria a circulação do ar para o seu interior, transformando a utilização desse EPI num verdadeiro "efeito-abafamento”. Se a temperatura externa estiver perto dos $40^{\circ} \mathrm{C}$, a temperatura no interior de um EPI será bastante elevada para um ser humano, podendo causar riscos à saúde.

Um trabalhador rural brasileiro chega a trabalhar mais de 12 horas por dia, seis vezes na semana em temperaturas externas que podem atingir $40^{\circ} \mathrm{C}$ em um verão bastante úmido, estando sujeito a uma condição de trabalho bastante insalubre, que pode trazer sérias conseqüências negativas à sua saúde.

Considerando as estimativas já apresentadas, de 20 minutos com EPI e 90 minutos com roupa normal, para um trabalhador em atividade moderada elevar a sua temperatura corporal em $1,5^{\circ} \mathrm{C}$, ter-se-ia que as condições ambientais de um trabalhador rural brasileiro, na realidade, ultrapassariam, em muito, essa faixa do conforto térmico, especialmente se este estiver utilizando o EPI na forma recomendada.

Desse modo, seria possível perceber a causa da resistência de alguns trabalhadores rurais ao uso de certos EPIs. Um motivo estaria na percepção do risco desses trabalhadores rurais de que poderiam estar trocando esse desconforto térmico certo, agudo e de curto prazo pelo risco de se contaminar, o que na maioria das vezes traria conseqüências incertas, crônicas e de longo prazo, as quais muitas vezes parecem ser assintomáticas e de difícil diagnóstico (VEIGA, 2007).

\section{A contaminação por agrotóxicos numa vinicultura francesa}

As reflexões apresentadas neste tópico dizem respeito à eficácia real dos EPIs, quando são utilizados em boas condições ou nas condições recomendadas. Uma pesquisa desenvolvida com base na abordagem da ergo-toxicologia (SZNELWAR, 1992; MOHAMMED-BRAHIM, 1996; GARRIGOU et al., 1998; MOHAMMED-BRAHIM et al., 2003) caracterizou a contaminação por agrotóxicos (carbamatos) dos viticultores franceses. Esta pesquisa identificou as formas de contaminação em função da natureza da atividade de trabalho e de seus determinantes, sejam eles de ordem técnica, humana ou organizacional (BALDI et al., 2002; BALDI et al., 2006)

Nessa pesquisa foram realizadas 96 jornadas de observação em situação real que permitiram produzir diferentes tipos de dados para cada etapa da atividade de trabalho (preparação, aplicação do produto e limpeza). A medida da contaminação foi realizada pela análise da quantidade de agrotóxico depositada sobre gazes cirúrgicas de $10 \mathrm{~cm}^{2}$ fixadas diretamente sobre a pele em diferentes segmentos corporais do viticultor (cabeça, mãos, troncos, braços e pernas), como pode ser visualizado no desenho esquemático da Figura 1. Essas gazes eram trocadas no final de cada fase da atividade.

Durante as observações, os viticultores realizaram as diferentes operações de acordo com seus hábitos normais. Alguns utilizavam equipamentos de proteção, outros não. Alguns equipamentos estavam em melhor estado que outros.

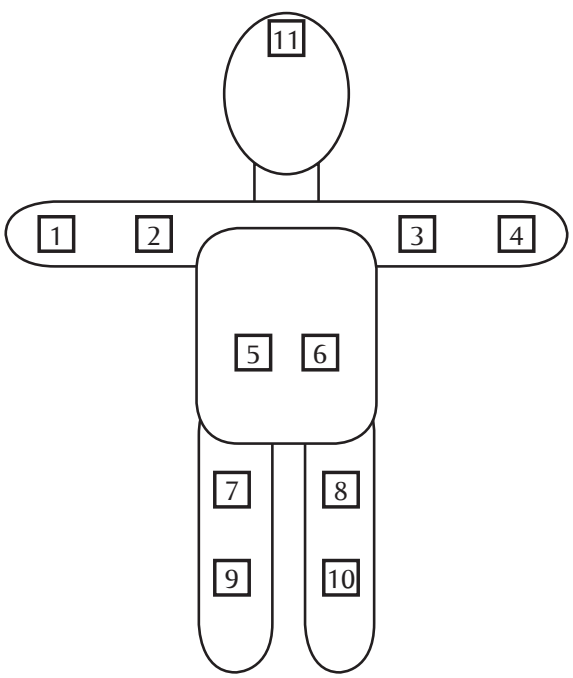

Figura 1 Desenho esquemático da localização dos pontos de coleta para a medição da contaminação cutânea 
Os resultados foram expressos em mg de matéria ativa depositada sobre a pele do agricultor (após análise da gaze colocada na superfície corporal considerada). A Tabela 1 apresenta a distribuição da contaminação (mínimo, percentil 25\%, mediana, percentil 75\%, máximo). A constatação mais surpreendente se deu pela comparação das distribuições dos valores de contaminação por pessoas que usavam o uniforme sobre as pessoas que não o usavam: pessoas "protegidas" poderiam apresentar contaminação até mais elevadas que pessoas "não protegidas". Com o tratamento estatístico dos dados, pôde-se constatar:

- o fato de utilizar uniforme de proteção não evita totalmente a contaminação;

- durante a etapa de preparação, a utilização do uniforme reduz a contaminação;

- durante as fases de aplicação do produto e de limpeza, as pessoas que utilizavam os uniformes de proteção eram globalmente mais contaminadas que aquelas que não utilizavam.

Estes resultados provocaram um forte questionamento junto às instituições francesas de prevenção, uma vez que uma das principais recomendações preconizadas era a utilização dos uniformes de proteção individual, em particular uniformes classificados como tipo $4^{7}$, ou seja, que protegem contra aerossóis.

A partir de numerosas discussões com diferentes especialistas da área, sindicatos franceses e europeus, foram propostas as seguintes hipóteses explicativas para esse tipo de contaminação:

- a pré-contaminação dos EPIs se explicaria pela sua reutilização: Quando esses EPIs são guardados em locais previamente contaminados, sendo pouco ou nada limpos, é provável que ocorra uma contaminação acumulativa no interior dos EPIs;
- a ineficácia dos EPIs em função das diferentes fases da atividade: Os equipamentos diminuem sua eficácia a cada etapa do processo que vai da preparação à limpeza. Na Tabela 1, somente na fase de preparação, a mediana com utilização do EPI é inferior à mediana sem o EPI. Nas demais fases da atividade (aplicação e limpeza), as medianas com a utilização do EPI são superiores às medianas sem EPI. Este dado indica uma contaminação maior em trabalhadores rurais utilizando EPIs.

- EPI e crença de proteção: Para certos viticultores, o fato de estar utilizando os EPIs poderia reforçar a crença de se sentir protegidos, o que poderia se traduzir na falta de atenção a certas formas básicas de precaução;

- práticas mais seguras desenvolvidas em função da experiência: Durante as observações, práticas muito distintas puderam ser caracterizadas, por exemplo, em relação ao cuidado para se abrir e derramar (virar) os sacos de agrotóxicos em pó. O fato de limitar a criação de nuvens de poeira, de derramar o saco em contato direto com a cuba se opunha a práticas em que abrir e derramar o conteúdo do saco se fazia bruscamente. A existência deste saber-fazer com prudência pode, em alguns casos, limitar fortemente a contaminação direta, assim como a contaminação indireta gerada pelo contato repetido de certas partes do corpo com materiais previamente contaminados.

Já no final de 2006, uma nova hipótese sobre a permeabilidade dos EPIs aos agrotóxicos foi formulada por Baldi et al. (2006) para explicar a ineficácia real dos EPIs recomendados pelas instituições de prevenção francesas. Nesse trabalho realizado em conjunto com um fabricante de agrotóxicos, foi analisada a permeabilidade dos uniformes de proteção utilizados com agrotóxicos. Face à toxicidade de um herbicida

Tabela 1 Contaminação externa em mg de matéria ativa depositada sobre a pele do agricultor

\begin{tabular}{|c|c|c|c|c|c|c|}
\hline \multirow[t]{2}{*}{ Distribuição } & \multicolumn{2}{|c|}{$\begin{array}{c}\text { Preparação } \\
\text { EPI }\end{array}$} & \multicolumn{2}{|c|}{$\begin{array}{c}\text { Aplicação } \\
\text { EPI }\end{array}$} & \multicolumn{2}{|c|}{$\begin{array}{c}\text { Limpeza } \\
\text { EPI }\end{array}$} \\
\hline & Sem & Com & Sem & Com & Sem & Com \\
\hline Mínimo & 0,6 & 0,1 & 0,3 & 0,3 & 0,2 & 0,2 \\
\hline Percentil 25\% & 2,2 & 0,7 & 2,2 & 2,8 & 1,4 & 1,9 \\
\hline Mediana & 4,2 & 3,2 & 4,0 & 7,7 & 2,3 & 9,3 \\
\hline Percentil 75\% & 8,8 & 8,3 & 18,8 & 21,6 & 10,6 & 28,9 \\
\hline Máximo & 23,7 & 564,6 & 73,9 & 463,1 & 54,4 & 151,6 \\
\hline Número de observações & 21 & 44 & 31 & 40 & 8 & 18 \\
\hline
\end{tabular}

Fonte: Baldi et al. (2002 e 2006)

\footnotetext{
${ }^{7}$ Em relação ao nível de proteção contra produtos químicos, os EPIs, mais especificamente os uniformes de proteção, podem ser classificados em 6 tipos (1 a 6) que definem o nível de proteção. Quanto menor o tipo, maior a proteção. Por exemplo, o tipo 1 protege contra gases, o tipo 3 protege contra líquidos, o tipo 4 protege contra aerossóis e o tipo 5 protege contra partículas. Fonte: http://www.ecocampus.net
} 
utilizado na cultura francesa de banana, entre outras, essa indústria química decidiu desenvolver um serviço de proteção associado à utilização desse produto. Numa primeira fase de desenvolvimento foi realizado, pelo Instituto Têxtil Francês (IFTH), uma série de testes de permeabilidade em diferentes tipos de uniformes recomendados para o uso com esse herbicida.

Os resultados desses testes foram alarmantes e colocaram em evidência a importância do fenômeno da permeabilidade na ineficácia dos EPIs. O produto puro (herbicida) depositado sobre o tecido de um EPI novo passou pelo tecido em um minuto (protocolo de teste EM 374-3 de 2004). Testes do mesmo tipo realizados com outros herbicidas do mesmo fabricante produziram resultados análogos. Um segundo teste com aerossóis dos mesmos produtos colocou em evidência que os mesmos penetraram através dos uniformes em menos de dez minutos.

Tais testes não foram repetidos para o agrotóxico utilizado no estudo de caso da viticultura francesa. Entretanto, por analogia, uma hipótese semelhante pode ser formulada. Na realidade, após reuniões realizadas com os fabricantes dos EPIs, pôde-se perceber que, em geral, a agricultura constitui um nicho, em termos de mercado mundial de EPIs inferior a $4 \%$. Nessas condições, os uniformes recomendados para a agricultura foram desenvolvidos para a indústria e "adaptados para a agricultura. Eles não teriam sido testados com a grande gama de compostos químicos utilizados nas formulações dos agrotóxicos para agricultura, o que explicaria o problema da permeabilidade dos EPIs. Essa constatação conduziu o fabricante do herbicida utilizado na cultura da banana a tentar desenvolver novos EPIs em conjunto com um pequeno fabricante brasileiro desses equipamentos de proteção.

Os autores do estudo consideram que na falta de maiores informações, em termos de precaução, poderse-ia recomendar EPIs dos tipos 2 ou 3 que, em teoria, ofereceriam mais proteção. Além disso, um nível mais elevado de proteção significaria um maior desconforto térmico, o que faria com que esses uniformes se tornassem totalmente inadequados à realidade do trabalho agrícola, uma das justificativas da não utilização de EPIs.

\section{Discussão}

A questão da concepção e do uso de EPIs pode ser considerada como um problema clássico de transferência de tecnologias (WISNER, 1997; PALIS et al., 2006). Eles foram concebidos para situações de utilização que correspondem muito pouco à realidade das condições de trabalho dos agricultores. A situação penosa do trabalho é uma realidade vivenciada que pode conduzir os agricultores a decidir limitar essa penosidade, em particular evitar o desconforto térmico e não se proteger.
Esses fatos colocam em evidência falhas do tipo organizacional ou latentes em relação à eficácia real dos EPIs. De acordo com Reason (2004), essas falhas seriam introduzidas nos sistemas por níveis hierárquicos superiores e estão relacionadas à gestão ou concepção dos sistemas produtivos. No caso dos EPIs, essas falhas estão relacionadas ao projeto, à certificação e à colocação desses equipamentos de proteção no mercado.

$\mathrm{Na}$ realidade, os dois casos aqui discutidos (brasileiro e francês) colocam em questão a ineficácia dos EPIs quando analisados em situações reais. Essas falhas do tipo organizacional têm implicação sobre a ação da maior parte das instituições competentes em termos de prevenção. Coloca-se em evidência a necessidade de normalizações sobre EPIs para a agricultura, o que já começa a ocorrer na comunidade européia, em particular na Alemanha (norma DIN 32781) e na Holanda. Esta é uma das tentativas de corrigir as falhas organizacionais.

A utilização de EPIs faz parte do dia-a-dia de muitos trabalhadores. Muitos projetos de Saúde e Segurança no Trabalho (SST) já contemplam, desde a sua fase de concepção, a utilização de EPIs. Sabe-se que os EPIs têm a finalidade de proteção, ou seja, reduzir/ controlar e não de evitar, os riscos à saúde e à segurança dos trabalhadores. Conseqüentemente, na maioria dos casos, a utilização de EPIs só deve ser considerada como um dos últimos recursos de tecnologia de proteção/controle de riscos aos trabalhadores (ILO, 2001).

As medidas de prevenção seriam aquelas que eliminam ou reduzem os riscos e perigos, atuando na sua fonte, ou seja, evitam e/ou reduzem a geração do risco ou do perigo. A prevenção deveria ser priorizada em relação a medidas de proteção ou atenuação dos riscos ou dos perigos. Na maioria das situações, "proteger" parece ser mais "econômico" do que prevenir.

Por isso, seria necessário que as legislações sobre Saúde e Segurança no Trabalho (SST) dispusessem de mecanismos que incentivassem medidas de prevenção por parte dos empregadores. Nesse tipo de legislação, deveriam, ainda, ser estimuladas medidas de proteção coletiva. Contudo, especialmente nas situações reais agrícolas, elas são pouco utilizadas pelas mais diversas razões, tais como custo elevado e por serem tecnologias pouco adaptadas às especificidades da agricultura.

Essa falta de estímulo para a adoção de medidas de prevenção como solução viável para controlar situações insalubres ou perigosas estimula a utilização dos EPIs como solução paliativa, uma vez que os EPIs, normalmente, parecem ser a solução economicamente mais vantajosa.

Um indicador dessa falta de incentivos para medidas de prevenção é a carência de estudos sobre desenvolvimento tecnológico de EPIs. O estado da técnica dos EPIs está bem distante da utilização desses equi- 
pamentos em situações reais. Grande parte dos EPIs não seria adequada à sua utilização e/ou finalidade. Na maioria das vezes, parece que os EPIs agrícolas não conseguem evitar a contaminação, o acidente ou a lesão, podendo ser, inclusive, como no caso dos estudos aqui discutidos, fonte de contaminação. A função do EPI passa a ser reduzir o risco ou mitigar a conseqüência, o que diverge da expectativa legal.

Essa valorização da proteção em detrimento da prevenção fica evidenciada quando se percebe que existe um número cada vez maior de projetos incompletos que já incorporam na sua concepção a utilização de EPIs para encobrir suas falhas. Em matéria de risco químico, em particular no que diz respeito ao risco de utilização dos agrotóxicos, as medidas de prevenção seriam mais recomendadas do que as medidas de proteção constantemente utilizadas (EPIs).

De fato, constata-se que a adoção de EPIs parece ser a solução mais utilizada para atender os requisitos legais. Em geral, eventuais responsabilizações penais ou civis colocariam no pólo passivo o agricultor e/ou o proprietário da plantação, muito embora a legislação brasileira estabeleça que, em matéria ambiental, a responsabilização seja solidária e objetiva, englobando todos os agentes envolvidos na cadeia produtiva, o que incluiria dos fabricantes de agrotóxicos aos trabalhadores rurais, passando pelos pontos de venda. Com isso, o agricultor tem a obrigação não só de fornecer o meio de proteção (EPI), mas também de garantir resultado (Lei 6938/81) ${ }^{8}$.

Parece existir, ainda, uma deficiência na concepção dos EPIs desde a sua fase de projeto. Grande parte dos EPIs é projetada para proteger contra agentes isolados, ignorando os potenciais efeitos sinérgicos. Outro aspecto que impacta diretamente na qualidade e na eficiência dos EPIs é o econômico. Normalmente, na escolha do EPI são consideradas variáveis financeiras, muitas vezes ignorando custos socioeconômicos (e.g. custos para saúde dos trabalhadores e custos de responsabilização trabalhista). A decisão de qual EPI utilizar é bastante influenciada pelo custo deste EPI (CROCKFORD, 1999).

Quando se examinam os problemas apresentados pelos EPIs nos casos estudados, as discrepâncias entre as tecnologias mais recentes e as práticas na proteção de riscos e perigos para a saúde humana são especialmente evidenciadas. A valorização técnica desses equipamentos como solução de proteção obriga que sejam produzidos e consumidos em massa. O EPI, enquanto tecnologia de proteção em saúde, deixa a desejar em diversos aspectos durante o seu ciclo de vida (do projeto até o descarte final), considerando-se o seu caráter complementar, a sua diversidade e sua escala de utilização.
Os EPIs utilizados nos casos estudados apresentam lacunas em suas funções como produtos que tensionam as organizações. Essas lacunas se desdobram em riscos e perigos adicionais aos pré-existentes (inerentes ao processo do trabalho) e estão associadas à concepção, ao uso, à manutenção e ao descarte dos EPIs, aumentando a complexidade da gestão da Saúde e Segurança no Trabalho (SST) nos casos em que os equipamentos são apresentados como solução de proteção. Para efeito de discussão, essas lacunas podem ser reunidas em dois grandes grupos: lacunas de concepção e lacunas de adequação aos processos de trabalho concretos (REASON, 2004).

\section{Lacunas de concepção dos EPIs}

Os EPIs são projetados para atenuar lesões aos usuários decorrentes da exposição a agentes específicos durante o processo de trabalho. A produção e a comercialização dos EPIs são autorizadas, tecnicamente, pela emissão de Certificados de Aprovação (NR-6). Essa autorização é amparada exclusivamente em ensaios laboratoriais relativos a agentes específicos, implicando uma orientação ao projeto de EPIs por agente. Assim, um protetor auricular é analisado no tocante ao isolamento acústico, um capacete, no tocante a choques e assim por diante. A legislação não requer validação do projeto dos EPIs em testes de campo que simulariam a sua utilização em condições reais e que facilmente evidenciariam deficiências provocadas por essa orientação especializada nos projetos dos EPIs.

As lacunas funcionais associadas à concepção decorreriam da adoção de metodologias desatualizadas de projeto de produto. Projetar EPIs para agentes isolados sugere a adoção de metodologias anteriores à década de 1970. Medeiros (1995) apresenta uma revisão de diversas metodologias de projeto de produto empregadas em diversos tipos de equipamentos. Um traço comum entre as metodologias revistas é o destaque para as análises dos processos de utilização e manutenção dos produtos como estudos necessários para a definição das especificações de projeto de quaisquer produtos. Se as metodologias de projeto empregadas estivessem atualizadas, as especificações dos produtos levariam em conta efeitos sinérgicos dos diversos agentes existentes nos contextos de uso, manutenção, armazenagem e descarte dos EPIs.

Caso essas análises fossem consideradas, os equipamentos teriam funções correspondentes às diversas situações reais de uso, manutenção e descarte. Destacam-se as lacunas relativas ao conjunto de agentes considerados nas especificações de projeto e as relativas às pessoas e aos métodos de trabalho adotados em seus respectivos contextos. Por exemplo, vários EPIs não parecem terem sido concebidos considerando os 
mais simples atos, e.g. vesti-los e despi-los. Ficou evidenciado que o trabalhador rural pode se contaminar em ambos os atos (BALDI et al., 2006).

As especificações dos EPIs enfatizam a proteção para agentes isolados e ignoram a coexistência de outros agentes nos processos de uso e manutenção, cuja importância é eventualmente agravada em decorrência do uso dos EPIs, como o desconforto térmico associado ao uso de roupas protetoras inadequadas em trabalhos a céu aberto em regiões de clima tropical, como visto nos estudos de casos (OLIVEIRA \& MACHADO NETO, 2005).

A partir dos resultados dos estudos apresentados neste artigo, pode-se constatar diversas falhas de projeto gritantes, como a permeabilidade dos tecidos empregados na confecção dos EPIs, roupas sem ventilação, criando um efeito-abafamento, exposição da pele decorrente da falta de análises antropométricas dos usuários na fase de concepção e a inevitável inadequação dos equipamentos, luvas de proteção com folgas excessivas e mangas de camisas que deixavam o punho descoberto.

Outras falhas também podem ser inferidas, tais como não haver disponibilidade no mercado de luvas de trabalho para crianças, embora crianças e adolescentes participem em diversos processos de trabalho nas atividades agrícolas dentro e fora das escolas, máscaras e protetores faciais que embaçam com a respiração, utilização exagerada de materiais sintéticos de elevado tempo de degradação ambiental (ciclo de vida) e degeneração dos EPIs ao longo da sua vida útil, como aumento da permeabilidade dos tecidos de roupas protetoras, que aumentam a cada lavagem. A informação sobre a redução da proteção dos EPIs com sua utilização, aumentando o risco de contaminação, não é divulgada pelos fabricantes aos compradores e usuários.

Na concepção da utilização em conjunto, EPIs como óculos de proteção e protetores auriculares são projetados para uso isolado, não integrado. As hastes dos óculos colidem com as conchas do protetor auricular, ocasionando que as funções de ambos os EPIs são prejudicadas.

\section{Lacunas de adequação dos EPIs aos processos de trabalho}

Além das dificuldades decorrentes do emprego de metodologias de projeto de produto obsoletas, as indicações para uso e manutenção dos EPIs atendem a sugestões de Serviços de Saúde e Segurança no Trabalho (SST) e a indicações em rótulos e manuais de instruções elaborados por fabricantes diversos, sem validação das indicações em testes de campo e sem referências para avaliação da adequação do estado do produto. EPIs são concebidos como produtos descartáveis, mas não são encontradas usualmente indica- ções de métodos de avaliação do fim da sua vida útil (MEDEIROS, 1995).

O uso de EPIs em regiões tropicais, especialmente na agricultura a céu aberto, agrava problemas de desconforto térmico. O uso do equipamento influencia na termorregulação, podendo dificultar a perda ou a acumulação de calor pelo corpo humano. Por isso, em atividades em que a utilização de EPIs seja essencial, existe uma probabilidade maior de problemas para saúde provocadas pelo calor (CROCKFORD, 1999).

Verificaram-se, nos casos estudados, restrições, por parte de vários trabalhadores rurais, ao uso dos EPIs. Constataram-se, inclusive, situações em que o uso dos EPIs aumentou a contaminação por agrotóxicos. Pressupôs-se que as deficiências de projeto e produção resultam em EPIs inadequados e desconfortáveis. Assim, as objeções dos trabalhadores rurais à utilização desses equipamentos encontram evidências empíricas e fundamentos concretos (BALDI et al., 2006).

As orientações sobre métodos de uso, manutenção e armazenagem dos EPIs não são validadas nos diversos tipos de situações de trabalho. Em conseqüência, os métodos prescritos não correspondem aos métodos praticados. Os rótulos e bulas dos agrotóxicos podem não apresentar uma boa legibilidade, com dimensões de letras inadequadas aos usuários. A redação também pode dificultar a comunicação das informações relevantes para o usuário, considerando-se a escolaridade da maioria dos trabalhadores rurais.

Normalmente, o projeto dos produtos não inclui aspectos relativos à sua manutenção. É difícil limpar resíduos presos ou absorvidos pelas linhas de costuras em aventais e outras roupas, assim como em dobradiças de óculos, dobras no interior de botas, luvas, protetores auriculares e assim por diante.

Não se encontram usualmente informações sobre manutenção, além da simples limpeza, em que o trabalhador também pode se contaminar. Além disso, a informação sobre manutenção não é adequada à realidade do usuário. A guarda de EPIs em uso e com manutenção inadequada facilita a contaminação dos armários e a dispersão de material contaminante entre os EPIs.

O descarte dos EPIs também não parece ser contemplado nos projetos. Os materiais empregados não são biodegradáveis nem biodestrutíveis. O descarte usual desses equipamentos como lixo comum sugere a ausência de avaliação do perigo representado pela concentração de materiais tóxicos neles presentes. Carecem de avaliação os efeitos decorrentes da adoção da queima de EPIs em fogueiras ou do enterro, constatadas no estudo de caso apresentado em Veiga et al. (2006).

Portanto, as lacunas funcionais no projeto, na concepção, no uso, na manutenção, no armazenamento e no descarte dos EPIs empregados nos estudos de caso analisados comprometeram a saúde humana, a qualidade da vida no trabalho rural e agravaram os problemas ambientais. A prevenção dos riscos e perigos 
à saúde humana e ao meio ambiente é um benefício e, como tal, tem custo. Se não resolvida no projeto do processo de trabalho, restam a proteção coletiva e a proteção individual. Mas é preciso que essa proteção seja eficaz, o que não aconteceu nos casos estudados.

\section{Considerações finais}

Estudos sobre EPIs, de modo geral, não enfatizam os problemas de projeto destes produtos. São estudadas sobretudo as contaminações sofridas pelos trabalhadores e pelo meio ambiente. Ainda há relatos sobre dificuldades na utilização dos equipamentos, destacando-se a valorização, pelo trabalhador, da percepção de desconforto imediato versus a percepção de riscos para a saúde em longo prazo.

A legislação brasileira é ingênua em relação aos EPIs quando aceita universalmente que o uso desses produtos deve eliminar ou neutralizar a insalubridade, assumindo que a proteção do trabalhador ao usar o EPI é eficiente. Nos casos estudados, a proteção parcial implicou em contaminação, mas a penalização dos responsáveis dependeria de uma fiscalização, que no Brasil está subdimensionada em relação ao número de casos.

As evidências encontradas mostraram que os locais de trabalho e o corpo dos trabalhadores foram contaminados, a despeito do uso de EPIs. Os EPIs não eliminaram nem neutralizaram a insalubridade, conforme estatui a legislação, e ainda agravaram a contaminação dos trabalhadores rurais em algumas atividades.

\section{Referências}

ABRASCO. Política nacional de ciência, tecnologia e inovação em saúde: uma proposta. Rio de Janeiro, abr. 2002.

AHASAN, R.; MOHIUDDIN, G.; FARUQUEE, S. M. Strenuous tasks in a hot climate: a case study. Work Study, v. 51, n. 4/5, p. 175-181, 2002.

BALDI, I. et al. Assessment of pesticide exposure in vineyard workers. In: CONFERENCE OF THE INTERNATIONAL SOCIETY OF EXPOSURE ANALYSIS/CONFERENCE OF THE INTERNATIONAL SOCIETY OF ENVIRONMENTAL EPIDEMIOLOGY, 12/14., 2002, Vancouver, BC, Canada. Epidemiology. Vancouver, 2002. abstract.

BALDI, I. et al. Pesticide contamination of workers in vineyards in France. Journal of Exposure Analysis and Environmental Epidemiology, v. 16, n. 2, p.115-24, 2006.

BERNON, J. Traitement du risque phytosanitaire à la MSA de l'Hérault. In: COLLOQUE DES CTR, CTN, CCMSA, 2002, Bagnolet. Anais..., Bagnolet, 2002.

BRASIL. Ministério Da Saúde. Proposta de Política Nacional de Ciência, Tecnologia e Inovação
Os EPIs utilizados nos casos estudados apresentaram problemas de projeto. Há uma grande discrepância entre o estado da técnica no projeto dos EPIs (melhores práticas) e os EPIs difundidos na agricultura, como os utilizados nos casos estudados. A tecnologia de produção desses equipamentos parece, também, carente de reformulação para que sejam produzidas proteções com qualidade mais próxima do estado da técnica a custos compatíveis com a realidade econômica dos produtores rurais, especialmente dos pequenos produtores.

Os equipamentos utilizados na agricultura, além de não protegerem integralmente o trabalhador, ainda agravaram os riscos e perigos, pois, nos casos estudados, tornaram-se fontes de contaminação por agrotóxicos. Os métodos de limpeza expuseram os trabalhadores rurais a contaminações imediatas, para aqueles que fizeram a limpeza, e mediatas, para aqueles que tiveram contato com a área onde foi realizada a limpeza. O trabalhador ainda se contaminou ao vestir e despir um EPI contaminado.

Portanto, a metodologia e a concepção utilizadas no projeto de EPIs devem ser reavaliadas, devendo ser estimulados estudos sobre desenvolvimento tecnológico nesses equipamentos. A possibilidade de contaminação do trabalhador rural dependerá da qualidade do EPI. Mantidos os projetos de produtos e as práticas atuais, as contaminações continuarão, produzindo efeitos agudos e crônicos à saúde e segurança ambiental e no trabalho. As legislações sobre o tema devem ser revistas para incluir mecanismos que incentivem a prevenção em detrimento da proteção.

em Saúde. Secretaria de Políticas em Saúde.

Departamento de Ciência e Tecnologia em Saúde, Dezembro 2002.

BRUNET, R. et al. Le risque et la parole: construire ensemble une prévention des risques du travail dans l'agriculture et l'industrie. France: Octares Editions, 2005.

COUTINHO, J. A. G. et al. uso de agrotóxicos no município de Pati do Alferes: um estudo de caso. Caderno de Geociências, n. 10, p. 23-31, 1994.

CROCKFORD, C. W. Protective clothing and heat stress: introduction. Ann. occup. Hyg., v. 43, n. 5. p. 287-288, 1999.

EDUARDO, M. B. P.; MIRANDA, I. C. S. Vigilância Sanitária. São Paulo: Faculdade de Saúde Pública da Universidade de São Paulo, 1998. v. 8, (Série Saúde \& Cidadania)

GARRIGOU, A.; MOHAMMED-BRAHIM, B.; DANIELLOU, F. Etude ergonomique sur les chantiers de déflocage d'amiante. Rapport final. OPPBTP/DRT. CT3. Bordeaux, 1998. 
HAVENITH, G. Heat balance when wearing protective clothin. Ann. occup. Hyg., v. 43, n. 5, p. 289-296, 1999.

ILO. International Labour Office. Guidelines on occupational safety and health management systems, ILO-OSH 2001, Geneva. 2001.

JOURDAN, M. Développement technique dans l'exploitation agricole et compétence de l'agriculteur. 167 p. Thèse (Doctorat en Ergonomie) - Laboratoire d'Ergonomie, Conservatoire National des Arts \& Métiers, Paris, 1989.

MEDEIROS, E. N. Análise de aspecto do gerenciamento do design de produtos em processos de modernização tecnológica sob o enfoque ergonômico. 286f. Tese (Doutorado em Engenharia de Produção) - Coordenação dos Programas de Pósgraduação de Engenharia, Universidade Federal do Rio de Janeiro, 1995.

MINAG. Ministère de l'agriculture et de la pêche. Note DGFAR/SDTE/N2006-5029. Analyse et synthèse des contrôles réalisés en 2003 et 2004 concernant le respect de la réglementation de protection de la santé, lors de l'utilisation des produits phytosanitaires au sein des entreprises agricoles. 2006

MOHAMMED-BRAHIM B. Du point de vue du travail ou comment sulfater la vigne autrement. Approche ergo-toxicologique des traitements phytosanitaires en viticulture. Mémoire de DESS d'ergonomie. France: Université Bordeaux 2, 1996.

MOHAMMED-BRAHIM, B. ; GARRIGOU, A. ; PASQUEREAU, P. Quelles formes d'analyse de l'activité de travail en ergotoxicologie? In: CONGRES DE LA SELF, 38., 2003, Paris. Actes... Paris, septembre 2003. p. 24-26.

NIEHS. National Institute of Environmental Health Sciences. Worker Education and Training Program. Safety awareness for responders to hurricane katrina: protecting yourself while helping others. 2005.

Available form: www.wetp.org. Access: 06 mar. 2006.

NIOSH. National Institute of Occupational Safety and Health. Protecting emergency responders: lessons learned from terrorist attacks. RAND publications. Brian A. Jackson, D. J. Peterson, James T. Bartis, Tom LaTourrette, Irene Brahmakulam, Ari Houser e Jerry Sollinger. 2002.

. Protecting emergency responders: community views of safety and health risks and personal protection needs. RAND publications. Tom La Tourrette, D. J. Peterson, James T. Bartis, Brian A. Jackson e Ari Houser. 2003.

. National Institute of Occupational Safety and Health. NIOSH's national personal protective technology laboratory: providing personal protective technology innovations for the 21st century. Strategic Plan. 2004.

. Protecting emergency responders: safety management in disaster and terrorism response. RAND publications. Brian A. Jackson, John C. Baker,
M. Susan Ridgely, James T. Bartis, e Herbert I. Linn. 2004a.

Protecting emergency responders: personal protective equipment guidelines for structural collapse events. RAND publications. Henry H. Willis, Nicholas G. Castle, Elizabeth M. Sloss e James T. Bartis. v. 4. 2006.

OLIVEIRA, M. L.; MACHADO NETO, J. G. Segurança na aplicação de agrotóxicos em cultura de batata em regiões montanhosas. Rev. bras. Saúde ocup., v. 30, n. 112, p. 15-25, 2005.

OPAS/OMS. Manual de vigilância da saúde de populações expostas a agrotóxicos. Brasília, 1996.

PALIS, F. G. et al. Our farmers at risks: behaviour and belief system in pesticide safety. Advance Access Publication, v. 28, n. 1, p. 43-48, 2006.

REASON, J. Managing the risks of organisational accidents. Ashgate, Hants, 2004.

RODAHL, K. Occupational Health Conditions in Extreme Environments. Ann. occup. Hyg., v. 47, n. 3. p. 241-252, 2003.

RUAS, A. C. Avaliação de conforto térmico: contribuição à aplicação prática das normas internacionais. São Paulo: Fundacentro, 2001. Conforto térmico nos ambientes de trabalho. São Paulo: Fundacentro, 1999.

SARGENT, $C$. Out of the Wreckage: Katrina. FireRescue Magazine. v. 23, issue 11, 2005.

STULL, J. O.; MACDOUGALL, K. D. After the storm: Katrina clean-up crews threatened by lack of personal protective apparel. Available from: www. kcprofessional.com. Access: 06 mar. 2006.

SZNELWAR L. Analyse ergonomique de l'exposition de travailleurs agricoles aux pesticides. Essai ergotoxicologique. Thèse (Doctorat en Ergonomie) - Laboratoire d'Ergonomie, Conservatoire National des Arts \& Métiers, Paris, 1992.

VEIGA, M. M. Agrotóxicos: eficiência econômica e injustiça socioambiental. Ciênc. saúde coletiva, Rio de Janeiro, v.12, n.1, p.145-152, 2007.

VEIGA, M. M.et al. Análise da contaminação dos sistemas hídricos por agrotóxicos numa pequena comunidade rural do Sudeste do Brasil. Cad. Saúde Pública, Rio de Janeiro. v. 1.22, n.11, p. 23912399, 2006.

WISNER, A. Ergotoxicologie dans les pays tropicaux. In: WISNER, A. et al. Anthropotechnologie, vers un monde industriel pluricentrique. Toulouse: Octares, 1997. p. 179-189.

WOOD, L. Heat Resistant. Occupational Health. v. 56, n. 7, p. 25-29,2004. 